\title{
Mobile Encounter Networks and Their Applications
}

\author{
Oleksiy Volovikov \\ University of Jyväskylä \\ Jyväskylä, FINLAND \\ volovikov@hotmail.com \\ Niko Kotilainen \\ University of Jyväskylä \\ Jyväskylä, FINLAND \\ niko.kotilainen@jyu.fi
}

\author{
Timo Juonoja \\ University of Jyväskylä \\ Jyväskylä, FINLAND \\ timo.juonoja@jyu.fi \\ Mikko Vapa \\ University of Jyväskylä \\ Jyväskylä, FINLAND \\ mikko.vapa@jyu fi
}

\author{
Matthieu Weber \\ University of Jyväskylä \\ Jyväskylä, FINLAND \\ mweber@mit.jyu.fi \\ Jarkko Vuori \\ University of Jyväskylä \\ Jyväskylä, FINLAND \\ jarkko.vuori@gmail.com
}

\begin{abstract}
This article presents Mobile Encounter Networks (MENs), which emerge when mobile devices come across each other and form a temporary connection between them using a common short-range radio technology. Local information exchanges between mobile devices results in a broadcast diffusion of information to other users of the network with a delay. Gasoline Price Comparison System (GPCS), which delivers newest gasoline prices to mobile users using mobile encounter information diffusion, is described as well as other application areas where MENs could be used. The feasibility of building MENs using Bluetooth is tested with the BlueCheese mobile encounter network middleware and it is found that the Bluetooth currently present in mobile phones does not adequately suit as a network technology for MENs.
\end{abstract}

Keywords- mobile encounter networks, Bluetooth, Symbian

\section{INTRODUCTION}

Mobile Peer-to-Peer (MP2P) networks are designed for resource sharing in a mobile environment. These networks include infrastructureless ad hoc networks as well as infrastructure based cellular networks with end terminals having capabilities to share their resources. There are various examples of peer-to-peer applications for ad hoc networks $[2,6,9,23]$, cellular networks $[1,13]$ or both $[3,8]$.

This paper presents a new class of mobile networks: mobile encounter networks (MENs) [10]. MENs do not require an infrastructure and do not have problems of multi-hop communication requiring much lower density of mobile devices compared to ad hoc networks for operation. There are however certain limitations of applications operating in MENs, but as will be shown, some applications are feasible to be built using the MEN architecture.

The paper is structured as follows. Section II presents MENs. Section III describes the BlueCheese mobile encounter network middleware and section IV the Gasoline Price Comparison System that uses the MEN architecture for information diffusion. Section V lists potential applications of MENs and the measurements are shown in Section VI. The paper is concluded with future work in Section VII.

\section{MOBILE ENCOUNTER NETWORKS}

MENs are formed of mobile devices coming across each other and having a connection between them using a shortrange radio technology (such as Bluetooth or 802.11 WLAN). One encounter contains the discovery of devices, connection establishment between two devices and the exchange of data. One mobile device can form connections to multiple other devices in succession. In this way, the information from one node can be copied to other mobile devices. The duration of the encounter is usually short, because of mobility of the devices, but it can also be long if the mobile devices are not moving. MEN is then the network resulting from all encounters. MENs are very dynamic, and in contrast to ad hoc networks, they do not provide continuous multi-hop communication, but only a successive pair-wise communication between two mobile devices.

Peer-to-peer (P2P) refers to decentralized and selforganizing overlay architectures of equal and autonomous entities. Peer-to-peer architectures allow finding and using of distributed resources and they do not usually have central entity, which manages the network. Mobile Peer-to-Peer then extends peer-to-peer by allowing resource sharing in a mobile environment. MENs are also used for resource sharing. They are decentralized and consist of equal and autonomous entities, but do not require functionalities for self-organization.

Multi-hop resource discovery commonly found in P2P systems is missing from MENs. Resource discovery in MENs is done via pair-wise communication between two mobile devices inside an encounter and does not involve other devices outside the encounter. The way of obtaining data in MENs is push-based rather than pull-based commonly found in other MP2P systems [19]. MENs are not intended for searching, but rather for spreading information to interested parties.

\section{A. Information Diffusion}

Information diffusion in MENs happens when a mobile device stores information obtained from another mobile 
device in an earlier encounter and later forwards the information to other mobile devices in new encounters. The diffusion of information in such a network is delayed and represents a way of replicating information lazily among the devices. The origin of the information diffused in MENs can be any external source e.g., user or device.

The devices participating in the mobile information diffusion need to provide some resources to the process. For example, transmitting information always requires some amount of battery power and therefore some part of the information diffused in the MEN needs to be relevant for the user of the device. However, in general participating in such a network is inexpensive, because transmitting information using short-range radio technologies does not involve a network operator and consequently payments are not needed.

Some devices in the network might restrict the diffusion of information by not forwarding any information. Usually, this reduces the speed of information diffusion in MENs, but in some cases it might be beneficial. For example in applications where any user can create content, a user could select which content is good and allow the further diffusion of that information to other mobile devices. Then, each mobile device when offered the same content multiple times decides using for example a threshold how many times the same content needs to be offered and if a given threshold is exceeded, the information is accepted. As a global effect, only content rated good enough would flow in the network. This is called collaborative filtering [21].

Earlier studies on information diffusion in a delay-tolerant networks using simulations can be found from [7,11,12,16,20,22].

\section{B. Benefits and Shortcomings}

Compared to infostations [5], MENs provide faster diffusion of information, because the mobile devices can obtain information not only from the infostation, but also from other devices.

In infrastructure based information diffusion, for example in a GSM network, the network is used to transmit information from a mobile device to a centralized server and mobile devices use this centralized server to obtain data. Compared to infrastructure based information diffusion, MENs often provide a slower information diffusion and limited coverage. This is because the information is only available to the mobile devices which have encountered other mobile devices providing the information. However, there are certain advantages in information diffusion over MENs. First, there is no need for infrastructure for transmission of data. Second, the information diffusion in MENs is inexpensive, because no external service provider is needed for the transmission of data. Also, because all communication happens inside encounters between two mobile devices, there is no need for an external server where information would be stored. Without an external server, which potentially could become a bottleneck in a large system, MENs are also very scalable.
MEN resembles an ad hoc network in the sense that it allows two mobile nodes that come within range of each other to establish a connection and exchange data. There are however many differences between MENs and what is usually considered as ad hoc networking.

Perkins [17] shows that the main problems in ad hoc networks is to provide multihop routing of data (in a unicast, multicast or broadcast way) through the mobile nodes which are potentially moving and continuously changing the configuration of the network. A route in an ad hoc network can be repetitively broken due to a node in its path moving out of reach of its neighbors and a significant research effort is put into designing algorithms for repairing broken routes without generating too much control traffic. Moreover, routing requires assigning global addresses to nodes, since the data sent by a source node is targeted to a specific node, which is not necessarily within the transmission range of the source.

Compared to ad hoc networks, MENs differ in that they do not provide any routing facilities, since the goal is to spread information to as many nodes as possible rather than target specific destinations: a source node running one given application over MEN sends data to any other node running the same application coming within its transmission range. The other node will cache the data, and later send it further when it comes within range of other nodes. There is no need for mechanisms preventing data to loop back to its original source as in many ad hoc networks protocols. Moreover, since the data is sent only to the neighbors which are within the transmission range, global addressing is not necessary (the underlying communication medium takes care of assigning addresses to the nodes which are within range of each other since the actual data transmission requires distinguishing different neighbors). In particular, MENs do not require the functionalities commonly found on the network layer of protocol stacks whereas the scope of ad hoc networks is mainly on the network layer. MENs operate on application layer and require from the protocol stack only unreliable link layer using some wireless radio technology and a reliable data transport functionality of transport layer (such as TCP).

\section{BLUECHEESE}

BlueCheese is a mobile encounter network middleware that is used between Bluetooth's transmission protocols and MEN applications. BlueCheese contains interfaces for MEN applications. The applications can connect to other devices and transfer data via BlueCheese. BlueCheese utilizes Bluetooth in data transmissions. L2CAP and RFCOMM are used for data transmissions and SDP for service discovery.

BlueCheese can only be connected to one other BlueCheese device at a time. Other devices are put into queue to wait until the prevailing connection is terminated. However several MEN applications may run on the connected devices and exchange data at the same time. 


\section{GASOLINE PRICE COMPARISON SYSTEM}

Gasoline Price Comparison System (GPCS) is a mobile encounter network application, executed in mobile devices with Symbian OS (Series 60). Its purpose is to help making decisions on where to refuel. To achieve this, a mobile device collects some attributes of each gas station where the user's car is refuelled and diffuses them to other mobile devices. These attributes are the brand and the location of a gas station, price and type of gasoline, and the time of buying gasoline.

The following scenario illustrates the use of the application. A driver, equipped with GPCS, buys gasoline at his/her favorite gas station. The attributes, described above, are sent into the application with a bill for the gasoline, bought by the driver's mobile device. As expected, in the near future mobile devices will have such possibility. Having received these attributes at the gas station, the mobile device starts diffusing them using a short-range radio technology to other mobile devices it encounters as the cars come across. After a certain period of time the application removes them automatically to prevent sharing and keeping outdated information. A threshold is used for this purpose. It is clear that the value of the threshold depends on the frequency of price changes at gas stations. In the same way, other drivers equipped with the GPCS, share their information about other gas stations. Making such exchanges, all participants receive information about further gas stations on their way, making it possible to choose the cheapest place where to refuel next time. This also boosts the market-based economy by giving customers equal information about the market situation.

\section{OTHER APPLICATIONS}

\section{A. Grocery Store Price Service}

The idea of the Grocery Store Price Service is very similar to GPCS. Having bought some goods at a grocery store, a user of such application gets a possibility to share their prices, time and location of the store with other users, (s)he encounters at the streets or other public places. Being aware of prices of goods taken from different grocery stores, the user can choose the cheapest place for shopping next time.

\section{B. Dating Service}

Every user of the Dating Service creates his or her profile of personal characteristics as well as a filter describing what characteristics are preferred for matching new friends. After that the user's mobile device is ready to share the profile with other devices it encounters as the users come across. Having received a profile from a paired device, both applications match their filters with the user profile instantly. In case of a match, the applications from both sides inform their users about it. The rest is up to them! As a reference implementation for Dating Service without information diffusion, see Nokia Sensor [15].

\section{Joke Service}

The users of the Joke Service can create new jokes and exchange jokes with other users encountered. Such application can provide the users a possibility to rate incoming jokes or even prevent their further propagation. This makes it possible to propagate only a subset of available jokes that the majority of users like while bad jokes will be quickly eliminated. A similar application is the tourist attraction service, where the users rate different tourist attractions, like restaurants, and via collaborative filtering the application can provide rated information about different tourist attractions. This application type can be realized for example with the Nokia Flier [14].

\section{Event Service}

In the Event Service, the initial content, for example tourist information, is obtained from an infostation, because such content is usually provided by commercial or state organizations. However, the utilization of MENs makes information diffusion much faster due to their ability to retransmit the content to other mobile devices. The same kind of mechanism could also be used for example to deliver Really Simple Syndication (RSS) Feed Services to users subscribed to certain RSS feeds.

\section{E. Newspaper Service}

Newspapers could also be distributed using MENs as described by Reti et al. [19] Short-range radio technologies are usually faster compared to infrastructure based networks like GSM/GPRS/UMTS networks and therefore large data files are more efficiently delivered using MENs. Also for the user, the delivery of newspaper would be cheap. To avoid piracy, the contents of the newspapers would be transferred using MENs, but the key for accessing an encrypted content could be obtained via a centralized server. With this kind of a mechanism the newspaper provider would be able to charge for the content.

\section{MEASUREMENTS}

We conducted a set of tests with the BlueCheese middleware to determine the feasibility of implementing mobile encounter networks on today's mobile device models having Bluetooth. The tests measured the power consumption of Bluetooth compared to power consumption without Bluetooth, Bluetooth connection establishment times and the time needed for discovering other Bluetooth enabled mobile devices. The tests were done using Nokia N-Gage, Nokia 6600 and Nokia 6630 mobile devices.

Bluetooth power consumption is a major factor in estimating the suitability of BlueCheese for MEN communications. Figure 1 illustrates Bluetooth power consumption compared to standby time. According to the graphs, a device with Bluetooth communication needs over 10 times greater power than the device in standby mode.

Measurements in Figure 2 show that device discovery takes about 15 seconds to complete regardless of the phone model. Search rate also depends on how many devices are around. Measurements illustrate that if the number of devices around grows by one, the device discovery time grows about $1-2$ seconds. 


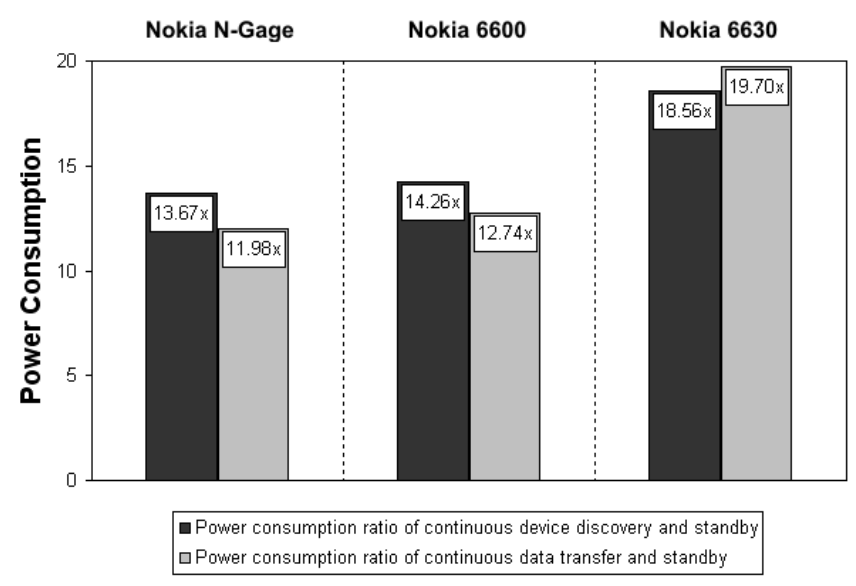

Figure 1. Bluetooth power consumption in contrast to standby time.

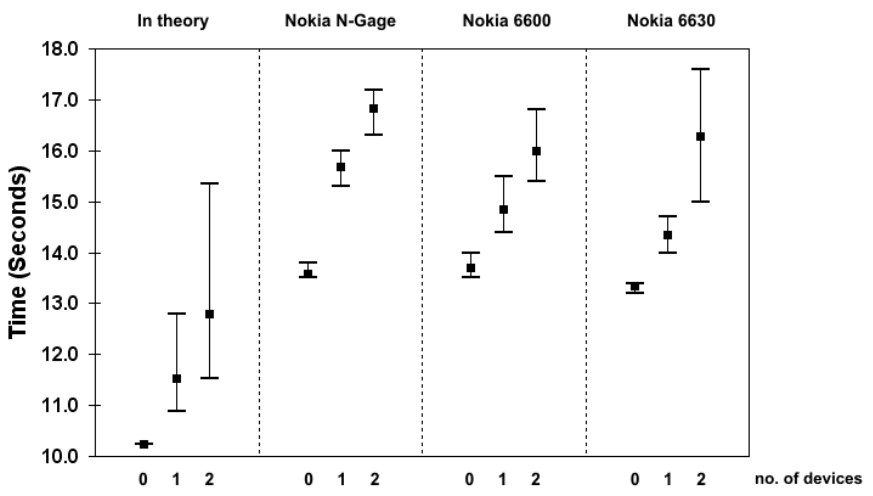

Figure 2. Min, max and mean of device discovery times with 0,1 and 2 devices around in theory and in practice.

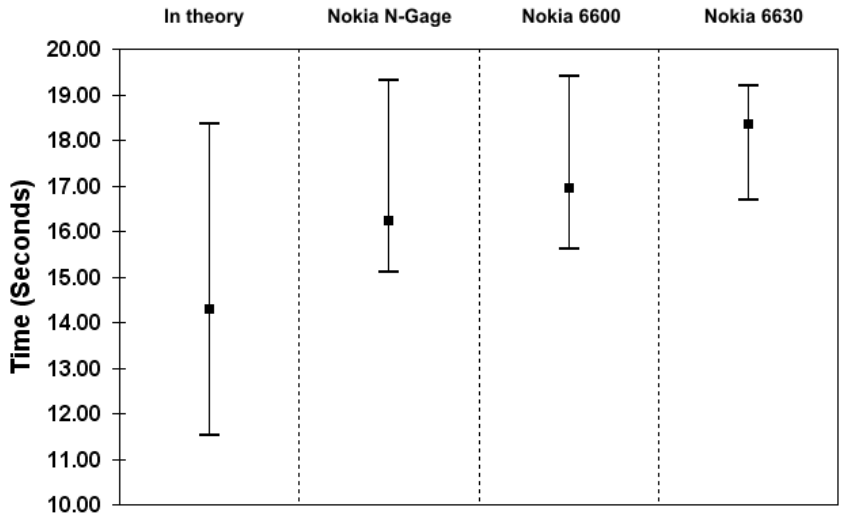

Figure 3. Min, max and mean of connection establishment times in theory and in practice.

The theoretical values were calculated. According to the Bluetooth 1.1 specification (in Nokia N-Gage and 6600), the device search takes 10.24 seconds in an error free environment. An enhanced inquiry requires 5 seconds and interlaced inquiry 2.5 seconds in Bluetooth 1.2 (in Nokia 6630), but 10 seconds is often used due to compatibility reasons.

Connection establishment rate was measured as a time from starting the Bluetooth inquiry to receiving a connection establishment event in BlueCheese. Figure 3 illustrates the connection establishment rate compared to theoretical values.
Time to establish a L2CAP connection is small compared to the time for device discovery. This lengthy discovery becomes critical in certain situations e.g., in peer communication wherein the devices are in motion. For this there are plenty of developed solutions to assist the device discovery. For example Radio Frequency Identification (RFID), Infrared Data Association (IrDA) and visual tags (a bar code for Bluetooth address) are used. These solutions are not decent for BlueCheese, because they assume that devices know the presence of each other whereas the key factor in BlueCheese is in information diffusion among unknown devices.

The main component in case of measuring and analysing BlueCheese is Bluetooth. MEN communication requires an advantageous combination of power consumption, availability, data transmission range and data transmission rate. Although Bluetooth has a low power consumption, the device search consumes too much power. If the device discovery is performed all the time, a full battery is exhausted within less than half of a day in all tested devices (Nokia N-Gage, Nokia 6600 and Nokia 6630). Because there are no low-powered low-level detectors for finding other Bluetooth devices, the device search has to be done manually at some intervals. If the interval is extended, the data spreading will be less efficient since there will be fewer connections whereas if the interval is decreased, the power consumption grows. Finding the optimal interval between device searches is very hard since it cannot be known when there is a considerable number of other Bluetooth devices around and especially devices with BlueCheese. There is a possibility of exhausting the battery without having discovered any device.

Availability depends on the latency of establishing the connection. Bluetooth device discovery lasts about 10-20 seconds depending on how many devices are around. This longish discovery time is critical when devices move during the discovery. For example, if one device is moving past a second device, the time required to perform the discovery may in fact exceed the time during which the two devices are in range of each other. This prevents communication between devices even if the device discovery runs non-stop.

The short range of Bluetooth with a lengthy discovery makes Bluetooth an unsatisfactory solution in a mobile encounter network environment. The device discovery in BlueCheese is performed by completing the procedure all the way. To enhance the BlueCheese device and service discovery, a condensed inquiry might be used as well, like for example halving the inquiry time. Then the probability of finding devices decreases, but the availability increases. Also a specific connection handshake protocol could be useful, because there are two connections in the connection establishment procedure: service search (SDP) and the actual data connection (L2CAP). Thus the time consuming connection establishment could be improved by creating only one connection instead of two.

Symbian OS development is also a problem in the construction of BlueCheese. For example, if two devices are 
within the data transmission range, the discovery fails if both devices discover concurrently because of the restriction of one Bluetooth activity at a time. Therefore it is suitable to prevent one device from discovering, if the other device is already discovering. This brings along the problem of implementation due to the dissimilarities of Symbian OS versions. In all Symbian OS versions it is still possible. However a solution that works in versions 6.1 and 7.0, is not compatible with Symbian OS version 8.0 even if it is more recent. That is the reason for the new Bluetooth Application Program Interface (BAPI), which defines a new entity for using Bluetooth functionalities. Bluetooth API entails several benefits but the API cannot be utilized due to the fact that it is not supported in older versions of devices. Therefore it is necessary to build a different version of the software for different Symbian OS.

Bluetooth as a transmission technique is not suitable for mobile encounter network communications on account of the reasons mentioned above. Bluetooth is suited for Client-Server based communication like for example multiplayer gaming over Bluetooth or a stable point-to-point connection such as the wireless headset providing hands-free audio.

\section{CONCLUSIONS AND FUTURE WORK}

Mobile Encounter Networks are emerging as a new area of mobile communication, because of wide-spread use of shortrange radio technologies in todays mobile devices. Some applications are well suited for MENs, which are restricted to only one-hop communication. Compared to ad hoc networks, simpler algorithms can be used, and compared to cellular network based MP2P applications, no infrastructure is needed.

However, current versions of Bluetooth have significant limitations affecting its usability for MEN applications. The main drawbacks are Bluetooth's power consumption for device discovery and the time needed for locating and establishing a connection with encountering devices.

As an alternative, ZigBee [24] looks promising for MENs. The key factor of ZigBee is low power consumption. Advances in low-power design have enabled battery life to be typically measured in years whereas the Bluetooth power consumption is measured in days. In transmit/receive mode ZigBee drains approximately twice less than Bluetooth.

Low latency is another important feature of ZigBee. CSMA-CA and beaconing bring in high throughput and low latency for devices. ZigBee devices can quickly attach, exchange information, detach, and then go to sleep to achieve a long battery life. Bluetooth devices require about 100 times more energy for this operation. The latencies of ZigBee and Bluetooth below show a significant difference in non-active slave operations. [3,18,24]

ZigBee is a swift network builder compared to Bluetooth. Bluetooth requires about 20 seconds for an inquiry which is not sufficient for efficient MEN communications. The same operation can be done in less than a second with ZigBee. Although ZigBee has a quadruple lower bandwidth, it is more suitable than Bluetooth for MENs because of its low power consumption and extremely low latency. The problem is the lack of support in contemporary devices.

$\begin{array}{lll} & \text { ZigBee } & \text { Bluetooth } \\ \text { New slave enumeration } & 30 \mathrm{~ms} & 20 \mathrm{~s} \\ \text { Sleeping slave changing to active } & 15 \mathrm{~ms} & 3 \mathrm{~s} \\ \text { Active slave channel access } & 15 \mathrm{~ms} & 2 \mathrm{~ms}\end{array}$

\section{REFERENCES}

[1] Andersen F.-U., de Meer H., Dedinski I., Kappler C., M“ader A., Oberender J. and Tutschku K., An Architecture Concept for Mobile P2P File Sharing Services, Workshop Proceedings of Informatik 2004 - Algorithms and Protocols for Efficient Peer-to-Peer Applications, pp. 229-233, 2004.

[2] Ding G. and Bhargava B., Peer-to-peer File-sharing over Mobile Ad hoc Networks, Proceedings of IEEE Annual Conference on Pervasive Computing and Communications Workshops, pp. 104-109, March 2004.

[3] Evans-Pughe C., Bzzzz zzz [ZigBee wireless standard], IEE Review, 49(3):28-31, 2003.

[4] Garg N., Shao Y., Ziskind E., Sobti S., Zheng F., Lai J., Krishnamurthy A. and Wand R., A Peer-to-Peer Mobile Storage System, Technical Report, TR-664-02, Computer Science Department, Princeton University, October 2002.

[5] Goodman D., Borras J., Mandayam N. and Yates R., INFOSTATIONS: A New System Model for Data and Messaging Services, Proceedings of the IEEE $47^{\text {th }}$ Vehicular Technology Conference, vol. 2, pp. 969-973, Phoenix, AZ, May 1997.

[6] Helal S., Desai N., Verma V. and Lee C., Konark. A Service Discovery and Delivery Protocol for Ad-Hoc Networks, Proceedings of the IEEE Wireless Communication and Networking Conference, New Orleans, LA, 2003.

[7] Jain S., Fall K. and Patra R., Routing in a Delay Tolerant Networking, Proceedings of the ACM SIGCOMM 2004, Portland, Oregon, August 2004.

[8] Kato T., Ishikawa N., Sumino H., Hjelm J., Yu Y. and Murakami S., A Platform and Applications for Mobile Peer-to-Peer Communications, http://www.research.att.com/_rjana/Takeshi Kato.pdf.

[9] Klemm A., Lindemann C. and Waldhorst O., A Special-Purpose Peer-to-Peer File Sharing System for Mobile Ad Hoc Networks, Proceedings of IEEE Semiannual Vehicular Technology Conference (VTC2003-Fall), Orlando, FL, October 2003.

[10] Kurhinen J., Korhonen V., Vapa M. and Weber M., Modelling Mobile Encounter Networks, $17^{\text {th }}$ Annual IEEE International Symposium on Personal, Indoor and Mobile Radio Communications, Helsinki, Finland, 2006.

[11] Kurhinen J. and Vuori J., Information Diffusion in a Single-Hop Mobile Peer-toPeer Network, Proceedings of the 10th IEEE Symposium on Computers and Communications, Cartagena, Spain, 2005

[12] Kurhinen J. and Vuori J., MP2P Network as an Information Diffusion Channel, Proceedings of the 62nd IEEE Vehicular Technology Conference, Dallas, 2005.

[13] Marossy K., Csucs G., Bakos B., Farkas L. and Nurminen J. Peer-to-peer content sharing in wireless networks. 15th IEEE International Symposium on Personal, Indoor and Mobile Radio Communications. Volume 1, pp. 109-114, 2004.

[14] Nokia Flier. http://europe.nokia.com/nokia/0, , 64488, 00. html

[15] Nokia Sensor. http://www.nokia.com/sensor/.

[16] Papadopouli M. and Schulzrinne H., Effects of power conservation, wireless coverage and cooperation on data dissemination among mobile devices, Proceedings of the 2nd ACM international symposium on Mobile ad hoc networking \& computing, Long Beach, California, 2001.

[17] Perkins C., editor. Ad Hoc Networking. Addison-Wesley, 2001.

[18] Poole I., What exactly is ... ZigBee?, IEEE Communications Engineer, 2(4):44-45, 2004.

[19] Reti T., Kortesniemi Y. and Välimäki M., Broadcasting Commercial Data on Mobile Peer-to-Peer Networks, Tokyo Roundtable 2002 Proceedings, Japan, 2002.

[20] Shah R., Roy S., Jain S. and Brunette W., Data MULEs: modeling a three-tier architecture for sparse sensor networks, Proceedings of the First IEEE InternationalWorkshop on Sensor Network Protocols and Applications, 2003.

[21] Terziyan V., Collaborative Filtering, Lecture Notes, http://www.cs.jyu.fi/ai/vagan/Collaborative Filtering.ppt

[22] Wolfson O., Xu B. and Sistla P., An Economic Model for Resource Exchange in Mobile Peer to Peer Networks, 16th International Conference on Scientific and Statistical Database Management, Santorini Island, Greece, 2004.

[23] Xue G.-T., Li M.-L., Deng Q.-N. and You J.-Y., Stable Group Model in Mobile Peer-to-Peer Media Streaming System, The 1st IEEE International Conference on Mobile Ad-hoc and Sensor Systems, Fort Lauderdale, Florida, USA, 2004.

[24] ZigBee Alliance, ZigBee Alliance - Wireless Control That Simply Works, http://www.zigbee.org, 2005. 